\title{
Endovascular Aortic Repair: The Renal Side of the Story
}

\author{
Mirko Menegolo, Francesco Squizzato, \\ Michele Piazza, Chiara Colacchio, Franco Grego, \\ and Michele Antonello
}

\subsection{Introduction}

It is common to find renal function modifications following abdominal aortic aneurysm (AAA) repair, but the precise short-term and long-term effects of open surgical repair (OSR) and endovascular repair (EVAR) on renal function are still unclear. This represents a largely debated topic, especially since the introduction of EVAR in the treatment of infrarenal AAAs with good early and midterm results, even for moderate- to high-risk patients $[1,2]$. The advantages of decreased morbidity, mortality, and length of stay $[3,4]$, in particular, justify the impressive wider use of EVAR in the last two decades; however, a stronger definition of longterm renal effects may help to better select patients for treatment with endovascular techniques.

Renal function impairment may occur after both open and endovascular repairs for several reasons. During open repair (OR), the main factor affecting renal function is the site of aortic cross-clamping, whether infra- or suprarenal, depending on the anatomical characteristics of

M. Menegolo $\cdot$ F. Squizzato $\cdot$ M. Piazza

C. Colacchio $\cdot$ F. Grego $(\bowtie) \cdot$ M. Antonello

Department of Cardiac, Thoracic and Vascular

Sciences, Vascular and Endovascular Surgery,

University of Padova, Padova, Italy

e-mail: franco.grego@unipd.it the aneurysm. Suprarenal clamping is in fact associated with hemodynamic changes that may cause postoperative renal dysfunction in up to $25 \%$ of patients [5]. Additionally, embolization into renal arteries or renal artery dissection may occur. On the other hand, EVAR still retains some intrinsic potential adverse effects on renal function due to endoluminal manipulation, which need iodine contrast dye (both for the treatment and the follow-up), and possibly secondary procedures. Moreover, since not all AAAs are amenable to infrarenal endovascular repair, in many cases, where the risky condition of the patient increases the operative mortality of traditional surgery, transrenal fixation (TRF) remains the only option for endovascular treatment $[6,7]$. The efficacy of the transrenally placed uncovered stent has been reported, but there are concerns about their effects on renal function. Several reports have indicated an acceptable short-term result of TRF on renal function [8-16]. Other reports seem to demonstrate a decrease of renal function after EVAR, regardless of the fixation level and especially in the long-term period [17-21].

Our experience in this field started more than 10 years ago, when we initially studied the shortterm effects of TRF on renal function in a series of patients treated with EVAR. More recently, our aim has been to compare OSR with EVAR 
(both with TRF and infrarenal fixation (IRF)) regarding long-term effects on renal function in the treatment of infrarenal AAAs.

All of our investigations have been characterized by the use of renal perfusion scintigraphy (RPS) to assess pre- and postoperative renal function. This choice derived from the fact that RPS has a significantly higher sensitivity than conventional serum markers, especially for demonstrating eventual worsening of function limited to a single kidney $[22,23]$.

\subsection{Renal Function Assessment}

Overall renal function is traditionally measured as renal clearance. The clearance for a specific compound is defined as the volume of plasma completely cleared of that compound per unit of time $\left(C_{\mathrm{s}}=U_{\mathrm{s}} \times V / P_{\mathrm{s}} ; C=\right.$ clearance, $U_{\mathrm{s}}=$ urinary output, $P_{\mathrm{s}}=$ plasma concentration). The clearance depends on all three fundamental renal functions: glomerular filtration, tubular secretion, and tubular reabsorption.

In clinical practice, creatinine clearance $\left(C_{\mathrm{Cr}}\right)$ is used as an estimate of the GFR (glomerular filtration rate), since creatinine is virtually neither secreted nor reabsorbed by renal tubules. In this situation, GFR results from the following formula: $\mathrm{GFR}=C_{\mathrm{Cr}}=U_{\mathrm{Cr}} \times V / P_{\mathrm{Cr}}$. From this concept, it appears that GFR can be estimated from the creatinine plasma concentration and creatinine urinary output in a given period of time, typically requiring $24 \mathrm{~h}$ urine collection. An even simpler method to estimate GFR consists in the $C_{\mathrm{Cr}}$ calculation based only on $P_{\mathrm{Cr}}$ measurement, using the Cockroft-Gault formula (GFR $=(140-$ age $) \times$ weight $(\mathrm{kg}) /\left(72 \times P_{\mathrm{Cr}}\right)$ with a correction factor of 0.85 to account in part for age-related differences for female patients). An alternative to the Cockroft-Gault formula is the Modification of Diet in Renal Disease (MDRD) study equation, $\mathrm{GFR}=175 \times\left(S_{\mathrm{Cr}}\right)^{-1.154} \times(\text { Age })^{-0.203} \times(0.742$ if female $) \times(1.212$ if African American $)$, which accounts for a standardized body surface instead of weight.
These methods of GFR estimation based on $P_{\mathrm{Cr}}$ (or $S_{\mathrm{Cr}}$, serum creatinine) have the advantage to be simple and readily available in all clinical settings but are limited in that they represent only an estimation of the true GFR that may depend on several other factors such as weight, muscle body mass, sex, age, and comorbidities. Furthermore, $P_{\mathrm{Cr}}$ results in a relatively lowsensitive diagnostic tool, since large changes on renal function must occur before a significant increase of $P_{\mathrm{Cr}}$, and, even if $\mathrm{CrCl}$ allows a more accurate evaluation of the glomerular filtration rate, this may be overestimated because of a variable tubular secretion of creatinine [23-25]. Another critical point is that $C_{\mathrm{Cr}}$ does not allow a renal function determination separately for each kidney, which may be useful, especially in a clinical research scenario.

Thus, a direct measurement of GFR should be advocated to obtain a more precise estimation of renal function. In this regard, RPS represents a more suitable and sensible test, since it evaluates separately the function of the kidneys and it is able to identify subtle renal functional loss [22].

For these reasons, in our studies, renal function has always been evaluated by RPS. This technique is based on the intravenous administration of a radiopharmaceutical and subsequent GFR calculation with the Gates method. For GFR determination, technetium-99m diethylenetriamine pentaacetic acid (DTPA) is typically used, since it is characterized by a negligible protein binding and it is only filtrated by glomerula without being secreted or reabsorbed by tubules. A decrease $\geq 20 \%$ of the GFR was considered clinically relevant. $S_{\mathrm{Cr}}$ was anyway tested as well, and $\mathrm{Cl}_{\mathrm{Cr}}$ was estimated at the same time of $S_{\mathrm{Cr}}$, with the Cockroft and Gault formula. Also in this case, postoperative change $\geq 20 \%$ above the baseline of $S_{\mathrm{Cr}}$ and/or $\mathrm{Cl}_{\mathrm{Cr}}$ was considered significant for renal dysfunction.

As an example of RPS potential, Fig. 6.1 reports the preoperative and follow-up RPS results in a patient treated with EVAR, with a postoperative decrease of GRF in the left kidney, without an overall decrease of $S_{\mathrm{Cr}}$. 

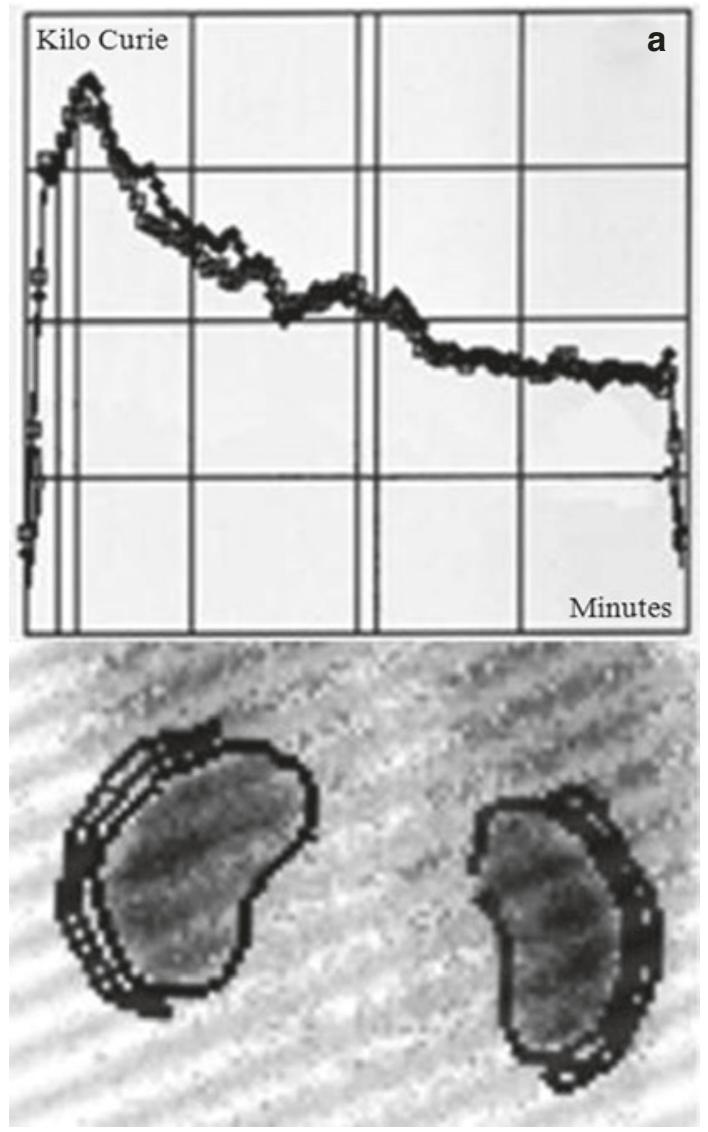

Fig. 6.1 Preoperative renal perfusion scintigraphy (RPS) using ${ }^{99} \mathrm{TC}-\mathrm{DTPA}$ (a). The postoperative RPS shows a decrease of glomerular filtration rate in the left kidney; in

\subsection{Causes of Renal Function Impairment After AAA Repair}

\subsubsection{Open Surgical Repair}

It is common to find a worsening in renal function after OSR of AAAs. This is mostly related to perioperative hypotension leading to acute tubular necrosis, which is more frequent in patients presenting with preoperative renal dysfunction or in patients treated in an urgent/emergent setting. Temporary renal artery occlusion (due to suprarenal aortic clamping), hypovolemic shock, post cross-clamp hypotension, and cardio-
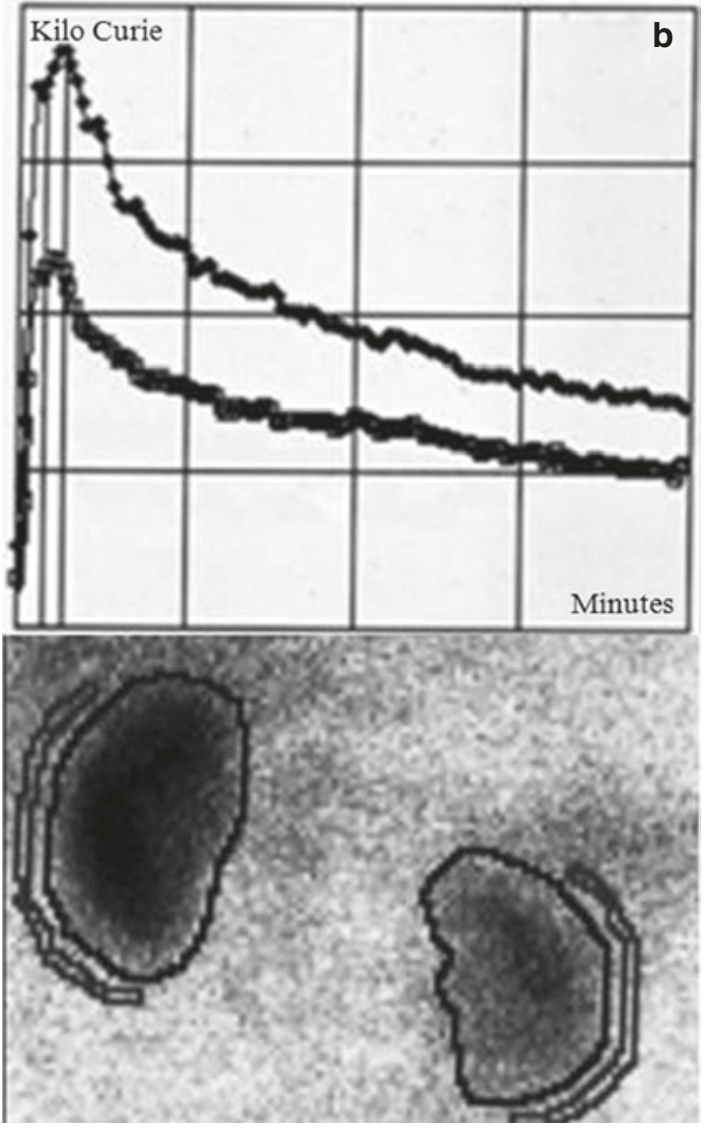

this patient no variation on total serum creatinine levels were observed (b)

genic shock in the perioperative period are the most common causes of acute renal dysfunction in open aortic surgery.

The site of aortic cross-clamping is another crucial factor [5], since suprarenal aortic crossclamping determines a temporary isolated period of renal ischemia, which can cause postoperative acute renal failure (ARF) in up to $25 \%$ of cases. Preoperative chronic renal insufficiency and the duration of cross-clamping are the most important predictors of ARF after suprarenal clamping. For this reason, the overall time of renal ischemia should generally be maintained at less than $40 \mathrm{~min}$, but the period of ischemia may be further prolonged, in particular in pararenal or suprarenal 
aneurysms requiring renal artery reconstruction. In these cases, a valid alternative consists in temporary renal reperfusion. In fact, in a previous study [24], we demonstrated that the temporary restoration of renal arterial inflow through a Pruitt-Inahara shunt for $3 \mathrm{~min}$ after $30 \mathrm{~min}$ of cross-clamping ischemia, protected from postoperative renal failure in juxtarenal aortic surgery, is also considered in patients at high risk for acute renal failure.

Another important cause of ARF in aortic surgery is renal atheroembolism. The quantity of microembolization produced during manipulation of the juxtarenal aorta during dissection depends on the embologenic potential of the atheromatous debris and the operative techniques used to prevent such an event. The clinical impact of renal microembolization depends on the quantity of functioning renal units before the procedure and the presence of other causes of ARF. In the absence of other factors favoring ARF and in the presence of normal mass of functioning nephron units, relatively large amounts of atheromatous microemboli can accumulate without immediate impact on renal function. In contrast, in patients with minimal renal reserve, the added insult of even minor microembolizations can lead to decompensation and ARF.

Another issue to consider during aortic OSR is the possible need for left renal vein ligation to gain proper exposure of the aortic neck. This maneuver is generally well tolerated if the operating surgeon takes care to preserve the principal tributaries of the left renal vein (the left gonadal vein, the left ureteral vein, capsular veins, lumbar veins and the ascending lumbar vein from above, and the left middle suprarenal vein and the inferior phrenic vein from below) performing the ligation near the confluence in the cava vein.

\subsubsection{Endovascular Repair}

Endovascular repair is generally considered a less invasive approach compared to OSR, but the early and long-term effects of EVAR on renal function are usually underestimated, since EVAR may also cause renal impairment through different mechanisms.

The main cause of renal function worsening after EVAR is represented by contrast-induced nephrotoxicity (CIN). This is defined as a $25 \%$ increase of the baseline serum creatinine or an absolute increase of at least $0.5 \mathrm{mg} / \mathrm{dL}$ (44.2 mmol/L) of serum creatinine, occurring between 24 and $72 \mathrm{~h}$ after contrast administration, and not imputable to other factors. The nephrotoxicity is caused by iodinated agents mediating vasoconstriction and tubular damage, and preexisting renal insufficiency, heart failure, diabetes, myeloma, and hypoproteinemia are the major risk factors for CIN. The volume of contrast media, contrast characteristics (including osmolarity, ionicity, molecular structure, and viscosity), and the route of administration (intra-arterial or intravenous) are also relevant. CIN is usually temporary, but in some cases, it may cause persistent renal insufficiency or lead to dialysis. Furthermore, EVAR is characterized by the need for strict follow-up, based on the repeated administration of contrast media for angio-CT scans, with a possible long-term renal function decrease. In this context, endovascular re-intervention performed for midterm and long-term complications (endoleaks, migration, limb occlusion, etc.) may also play a role.

To prevent contrast nephropathy, we administered $\mathrm{N}$-acetylcysteine $600 \mathrm{mg}$ orally twice a day for 1 day before and 2 days after EVAR and for any other contrast study during the follow-up period. Furthermore, all patients undergoing angiography or contrast-enhanced computed tomography (CT) receive hydration as guidelines recommend, and all nephrotoxic drugs (angiotensin-converting enzyme inhibitors, angiotensin receptor blockers, nonsteroidal anti-inflammatory drugs, and aminoglycosides) were withdrawn $24 \mathrm{~h}$ before contrast exposure [26]. In patients with post-procedural renal dysfunction (GFR $<65 \mathrm{~mL} / \mathrm{min}$ in men and $<60 \mathrm{~mL} / \mathrm{min}$ in women), the post-EVAR surveillance was usually based on non-contrast-enhanced CT to assess the sac size and the correct device position in duplex ultrasound scanning. 
Atheroembolism involving renal arteries may also occur during EVAR, due to manipulation of the juxtarenal aorta with guidewires and catheters. Regarding the clinical consequences of renal atheroembolism, the same considerations already described for OSR are valid.

Another matter to consider is the use of endografts with suprarenal fixation, which has been associated with the concern of short- and longterm renal function worsening due to atheroembolism and renal flow modifications induced by the presence of the free-flow proximal stent through the renal ostia.

An additional issue regards the use of endovascular techniques for the treatment of juxtarenal and pararenal aortic aneurysms, requiring the use of techniques involving the use of renal stents (chimney technique, fenestrated and branched endografts). In these cases, the risk of CIN is increased by the higher dose of contrast needed for the procedure, atheroembolism is increased by the need to cannulate one or both renal arteries, and there is a low but non-negligible risk of stent occlusion during the follow-up.

\subsection{The Effect of Transrenal Fixation on Renal Function: Results of a Previous Pilot Study}

Our first experience derived from the concern that TRF of abdominal endografts could result in potential renal function impairment due to embolization and flow modifications. Thus, we initially aimed to compare TRF to IRF focusing on the short-term effects on renal function in a cohort of 135 patients treated for infrarenal AAA. Between April 1999 and May 2002, 47 patients $(34.8 \%)$ had a TRF [25], while 88 had IRF. Indications for TRF included short $(1.5 \mathrm{~cm}$, ranging from 0.8 to $1.5 \mathrm{~cm})$ infrarenal proximal neck ( 28 patients, $59.5 \%$ ), angulation $<120^{\circ}$ between the flow axis of the proximal neck and the body of the aneurysm or a pyramidal-shaped proximal neck (11 patients, $23.4 \%$ ), and the presence of juxtarenal heavy $(50 \%$ of the circumference) calcification or thrombus ( 8 patients, $17.1 \%$ ). The endovascular procedure was always performed in the operating room under general or local anesthesia, and the contrast dye was usually $150 \mathrm{~mL}$ (range, 100$160 \mathrm{~mL} ; \quad$ Omnipaque-350). RPS (99m TC-DTPA) was performed preoperatively on the third postoperative day and repeated at 1 month in patients with worsening of renal function, defined as a decrease of GFR higher than $20 \%$.

The midterm follow-up included routine blood tests (with $S_{\mathrm{Cr}}$ ) at 3, 6, 12, 18, and 24 months and then yearly and RPS and contrast-enhanced CT scan at 6,12 months, and yearly thereafter.

In 15 patients $(31.9 \%)$, a change between the pre- and postoperative RPS was observed; it was permanent in 12 and transient in 3 cases. The permanent changes at RPS were expected in seven patients and unexpected in five. The expected changes were secondary to a segmentary renal infarction $(<25 \%$ of total renal parenchyma) caused by the planned exclusion of a renal polar artery. Unexpected changes were observed in five patients $(10.6 \%)$ at the RPS performed postoperatively; three of these patients had a slight preoperative renal insufficiency; and in three of these, a single kidney was involved.

The results of this study strengthened the hypothesis that $S_{\mathrm{Cr}}$ is not an accurate index to detect renal impairment since in three $(6.3 \%)$ patients a significant reduction of GFR (>20\%) was observed in the absence of any relevant change of $S_{\mathrm{Cr}}$ level. Moreover, the RPS revealed that the impairment of renal function was limited to a single kidney in two patients and bilateral in one. There were no statistical differences in terms of procedural and renal function results between TRF and IRF, but this may be related to the small number of patients (47) in this study.

However, this study showed that some patients are at risk of GFR impairment $>20 \%$ after EVAR, which can be demonstrated by RPS in $10.6 \%$ of cases, and this should be taken into consideration when selecting patients undergoing this procedure, especially in those with preoperative renal insufficiency. 


\subsection{The Effects on Renal Function of Open and Endovascular Aortic Repair: Long-Term Results}

The previous study was limited by the retrospective design, the low number of patients, and the short follow-up limited to early results. However, a comparison of EVAR with OSR would be appropriate in helping to direct patients to the best type of treatment. We then designed a prospective study, conducted from January 2003 to December 2007 [27], to compare OSR with EVAR over a long-term period. The hypotheses of the study were that patients undergoing OSR would demonstrate a lesser decline in renal function over time compared with those undergoing EVAR. In addition, we hypothesized that there would be lower renal function impairment after EVAR with IRF than with TRF. The presence of factors that could affect postprocedural renal function was considered as exclusion criteria: high preoperative renal dysfunction, renal artery stenosis $>60 \%$, renal accessory artery planned to be covered by the endograft, a single functioning kidney, hemodialysis and kidney transplant, and pararenal aneurysm requiring suprarenal aortic cross-clamping (Fig. 6.2).

Treatment selection was based on anatomical characteristics and patients' surgical risk. Preoperative angio-CT scan was acquired for all patients.

OSR was offered to patients with unsuitable anatomy, including proximal infrarenal aortic neck of less than $15 \mathrm{~mm}$ long or greater than
Fig. 6.2 Open surgical (OSR) versus endovascular repair (EVAR) for long-term investigation on renal function. In this study we excluded patients with creatinine clearance $<65 \mathrm{~mL} / \mathrm{min}$ in men and $<60 \mathrm{~mL} / \mathrm{min}$ in women; renal artery stenosis $>60 \%$; renal accessory artery planned to be covered by the endograft; single functioning kidney; hemodialysis; kidney transplant; and pararenal aneurysm requiring a suprarenal aortic cross-clamping. Open repair was offered in patients with unsuitable anatomy for EVAR

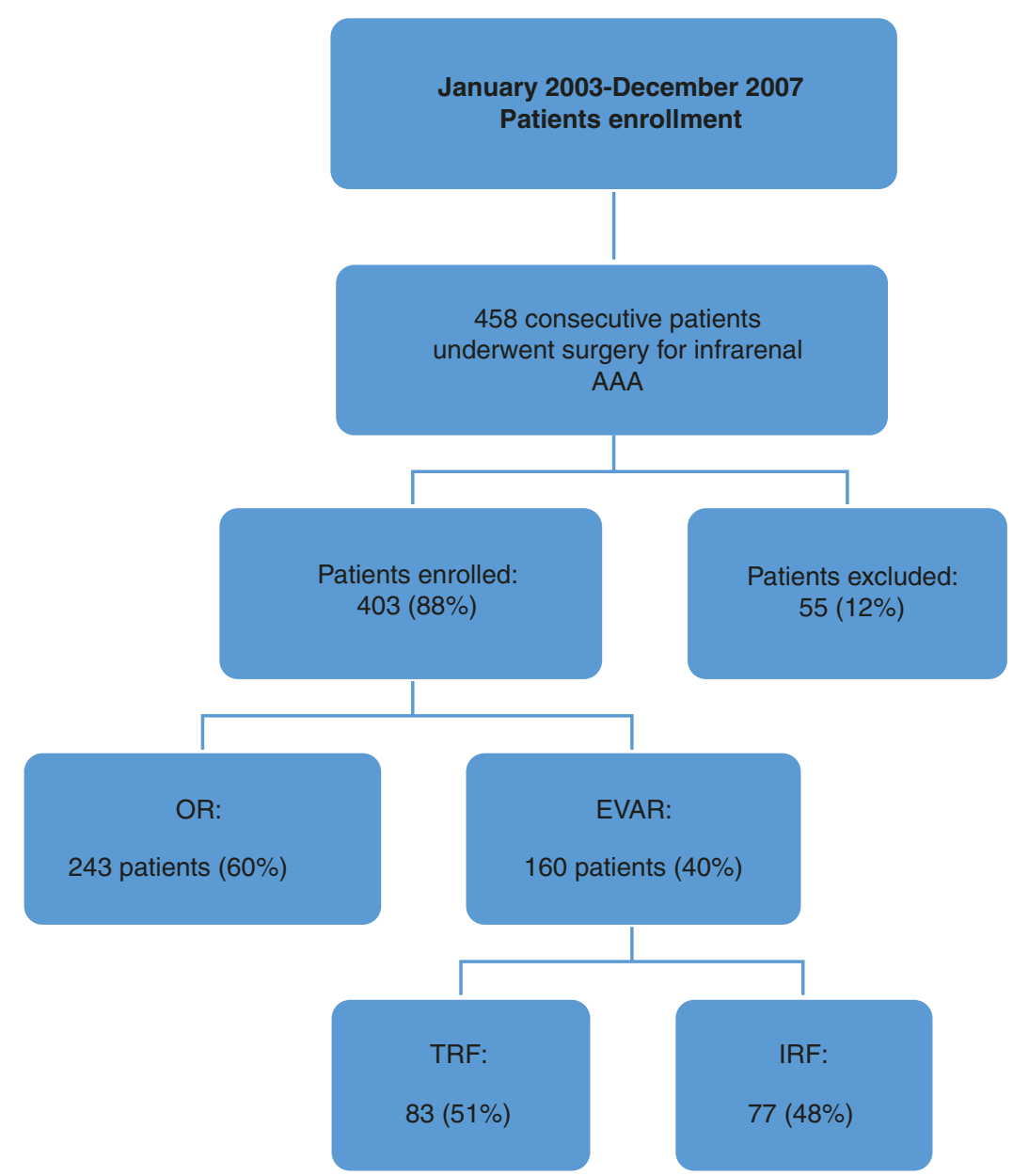


$30 \mathrm{~mm}$ in diameter, proximal neck angulation of greater than $60^{\circ}$, extensive neck thrombus, access vessel calcifications (more than $50 \%$ circumference involvement) with tortuosity (angulation $>60 \%$ ), common iliac or hypogastric arteries aneurysms of greater than $30 \mathrm{~mm}$ in diameter, and associated iliac occlusive disease. OSR was also offered as the first option in patients with long life expectancy, based on age and the absence of significant comorbidities.

The endovascular procedure was always performed in the operating room under general or peridural anesthesia; an intraoperative digital angiography was performed after the device deployment to identify any endoleaks and assess the patency of renal arteries.

Clinical and technical success was defined according to the reporting standards for endovascular repair [28].

RPS was performed preoperatively and postoperatively at 30 days, at 6 and 12 months, and then yearly. Patients that underwent EVAR underwent postoperative contrast-enhanced CT scan at 6 months, 12 months, and annually thereafter; in the OR group, follow-up was based on an annual ultrasound duplex scan.

During the study period, 458 consecutive patients were treated for infrarenal AAA. Fiftyfive patients $(12.1 \%)$ did not meet the inclusion criteria (Fig. 6.2). Among the patients enrolled, 160 (40\%) underwent EVAR; 77 had an IRF (Excluder; W.L. Gore \& Associates, Flagstaff, Ariz); and 83 had a TRF (Zenith; Cook Inc., Indianapolis, Ind). Of the 243 patients (60\%) treated by OSR, $126(52 \%)$ received a bifurcated aortoiliac graft, 98 (40\%) received tubular aortoaortic graft, and the remaining $19(8 \%)$ received bifurcated aortofemoral grafts. The mean infrarenal aortic cross-clamping time was $15.4 \pm 5.6 \mathrm{~min}$. No statistical differences were observed between groups for demographics and risk factors, classified according to the Society for Vascular Surgery/International Society for Cardiovascular Surgery reporting standards [27], except for the American Society of Anesthesiologists (ASA) score, coronary artery, and chronic pulmonary occlusive disease which were significantly higher in the EVAR group $(P<0.0001$ and $P=0.003)$.
In the open repair group, nine (4\%) patients died in the early postoperative period, four for myocardial infarction, three after a sigmoid infarction, and two for major arrhythmia.

Endograft deployment was successful in all cases. Two cases (1\%) required in the early postoperative period a femoro-femoral crossover for branch occlusion. The angio-CT scan performed after 30 days from the procedure showed the presence of a type II endoleak in 25 cases (15\%). No device migration was observed and there were no perioperative deaths.

A significant statistical difference emerged between OR and EVAR for early postoperative death ( $4 \%$ vs $0 \%, P=0.01)$. Follow-up ranged from 54 to 126 months (mean, 76 months) for OR and from 54 to 124 months (mean, 74 months) for $\operatorname{EVAR}(P=\mathrm{NS})$.

\subsubsection{TRF Versus IRF: Effects on Renal Function After EVAR}

The mean contrast dye volume per intervention was similar in the two groups (TRF $154 \mathrm{~mL} \pm 11$, IRF $148 \mathrm{~mL} \pm 10 ; P>0.05)$. No significant changes were observed for mean $S_{\mathrm{Cr}}$ between the preoperative and the postoperative periods (fourth day) both in the TRF group $(1.1 \pm 0.4$ to $1.2 \pm 0.4)$ and in the IRF group $(1.1 \pm 0.2$ to $1.0 \pm 0.4)$. Similarly, mean $\mathrm{Cl}_{\mathrm{Cr}}$ values remained stable in both groups $(60.1 \pm 13.8$ to $59.1 \pm 17.1$ for $\mathrm{TRF}$ and $59.8 \pm 12.9$ to $61.1 \pm 17.6$ for IRF).

No statistical differences were observed between the mean GFR measured at the RPS with the Gates method preoperatively and in the 30th postoperative day in both groups $(70.5 \pm 21.2$ to $69.5 \pm 23.1$ for TRF and $72.3 \pm 18.3$ to $70.7 \pm 20.2$ for IRF) (Fig. 6.3). Through analyzing the preoperative and postoperative value of the GFR separately, a significant change $(\geq 20 \%)$ emerged in 18 patients (11.2\%), 8 (9.6\%) from the TRF group and $10(12.9 \%)$ from the IRF group. In seven patients (4.3\%, 3 TRF; 4 IRF), a decrease of the GFR was limited to a single kidney; both kidneys were involved in the remaining 11 patients. 


\subsubsection{OSR Versus EVAR: Long-Term Results}

Mean $\mathrm{Cl}_{\mathrm{Cr}}$ at the RPS did not significantly change between pre- and postoperative (fourth postoperative day) values in both EVAR and OSR groups. Otherwise, analyzing the results separately for each patient, a significantly higher number of patients developed a $>20 \%$ GFR decrease in the EVAR group (EVAR 14\% vs OSR 4\%, $P<0.001$ ). Interestingly, the GFR impairment was limited to a single kidney in nine patients of the EVAR group.

A deterioration of the GFR was observed in the first 6 postoperative months in both groups, and this effect was more relevant in the EVAR group $(P<0.001)$. In the OSR group, there was no correlation between the type of aortic reconstruction, the time of aortic cross-clamping, and the early decline of renal function. In the EVAR patients, the GFR decreased gradually until $11 \%$ over the baseline at 9 years of follow-up (Fig. 6.4). In the OSR group, after the initial postoperative decline (6\% with respect to the baseline), there was an almost complete normalization of the GFR at 48 months (decrease of $2 \%$ ) and a reduction at 9 years of $3 \%$ (Fig. 6.4).

\subsubsection{Effect of Secondary Procedures on Renal Function in Patients Treated with EVAR}

Considering the long-term changes in renal function in patients who underwent EVAR, we aimed to identify who was at increased risk of GFR decrease. Our hypothesis was that patients needing secondary procedures had greater renal function impairment due to the repeated contrast dye administration for endovascular secondary procedures and for CT scans. At least one secondary procedure was performed in $17 \%$ of patients undergoing EVAR and in 3\% of patients undergoing OR $(P<0.0001$, Fig. 6.5). During the followup, the decline of GFR was remarkably higher in the subgroup needing additional procedures, with a reduction at 9 years of $14 \%$ with respect to $7 \%$ $(P<0.005$; Fig. 6.6). In this subgroup of patients, the mean volume of contrast dye used to perform the secondary procedure and the subsequent control angio-CT at 30 days was $223 \pm 36 \mathrm{~mL}$.

Furthermore, when comparing the GFR between OR and EVAR patients who did not require a secondary procedure, a significant difference was still present $(P<0.0005)$.

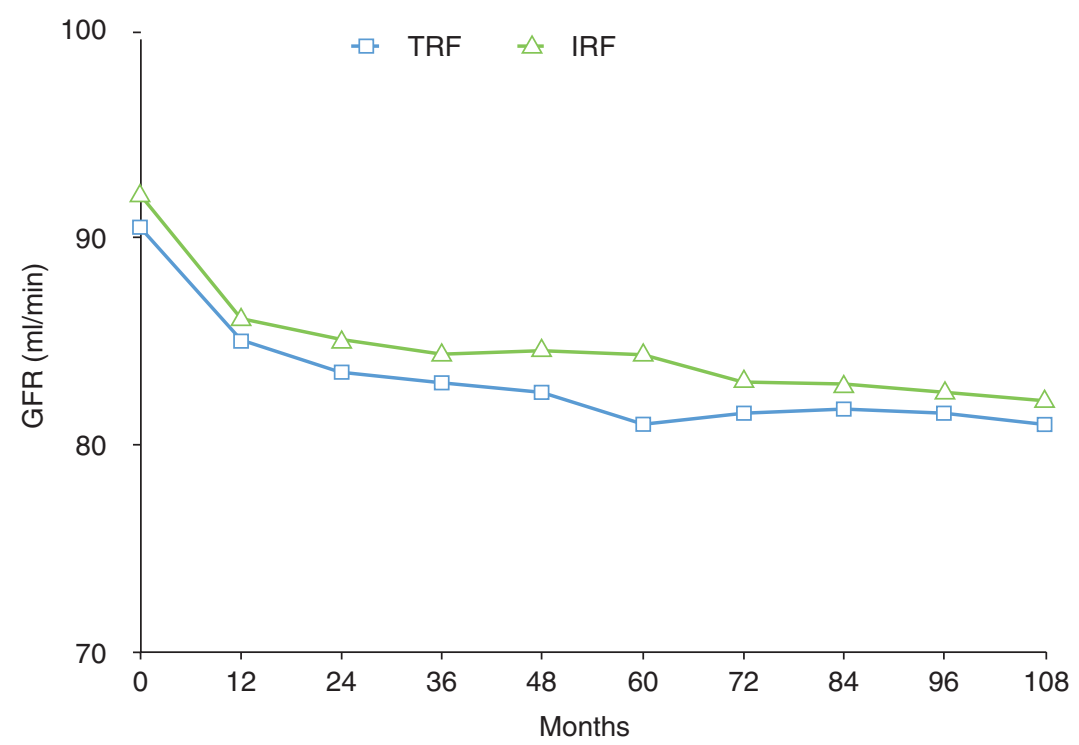

Fig. 6.3 Long-term results on renal function between patient undergoing endovascular aortic repair (EVAR) with transrenal fixation (TRF) and infrarenal fixation
(IRF). Glomerular filtration rate (GFR) was estimated using renal perfusion scintigraphy (RPS) 


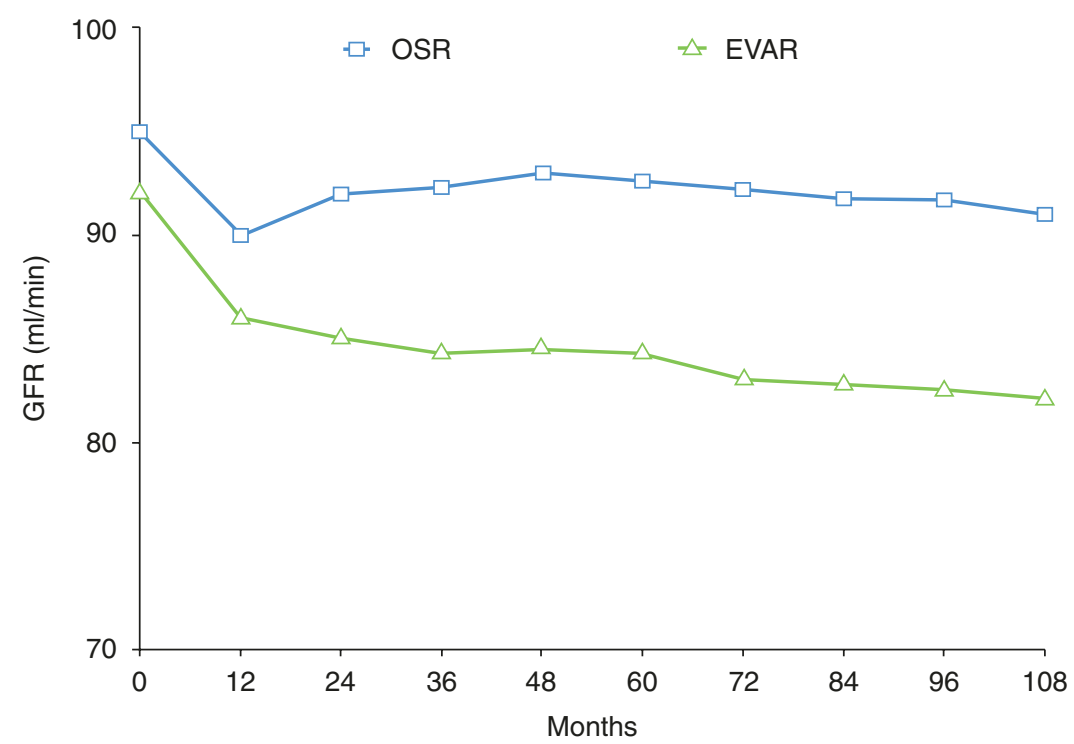

Fig. 6.4 Effects on renal function of open surgical repair (OSR) versus endograft repair (EVAR). Glomerular filtration rate (GFR) was estimated through renal perfusion scintigraphy (RPS). After an initial similar GFR decrease in both groups, a gradual normalization of GFR in the OSR group and a progressive worsening in the EVAR group were observed

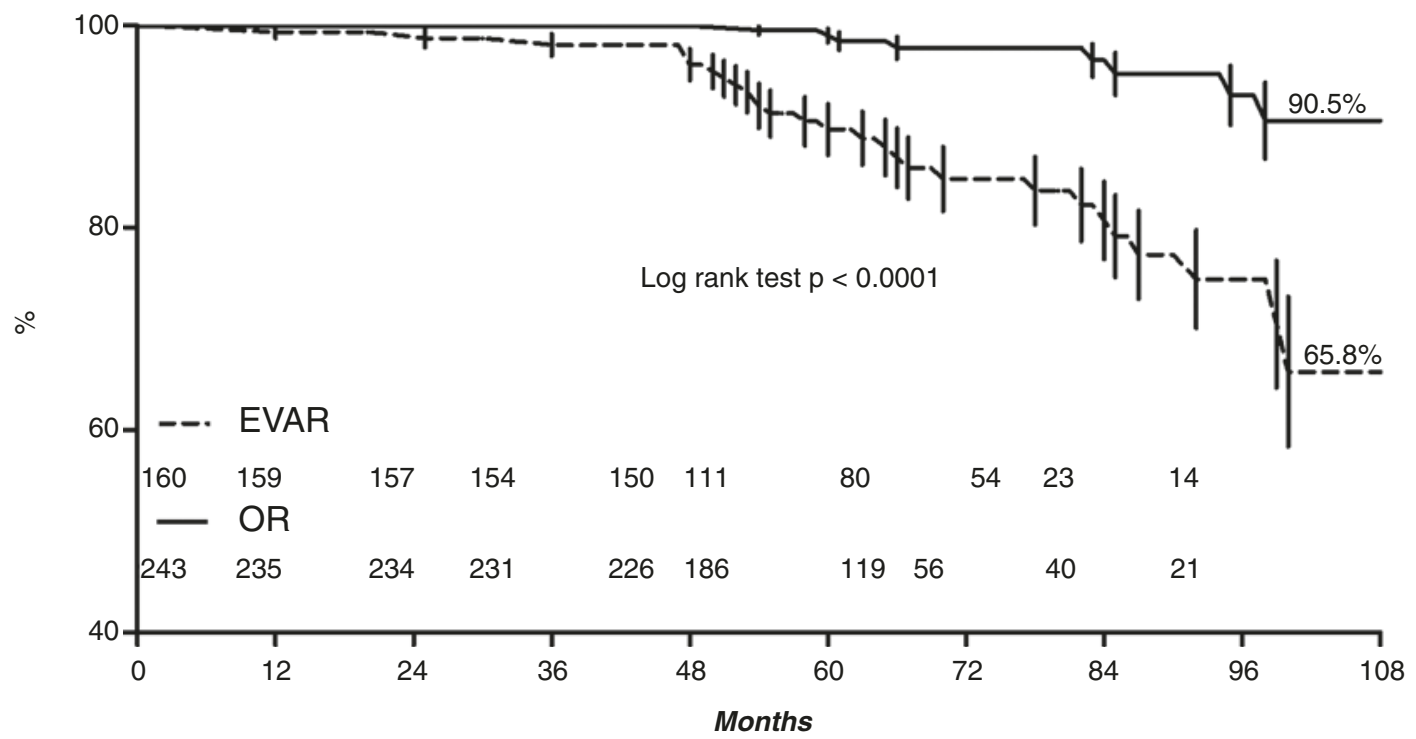

Fig. 6.5 Freedom from secondary procedure after open surgical repair (OSR) and endovascular aneurysm repair (EVAR) 


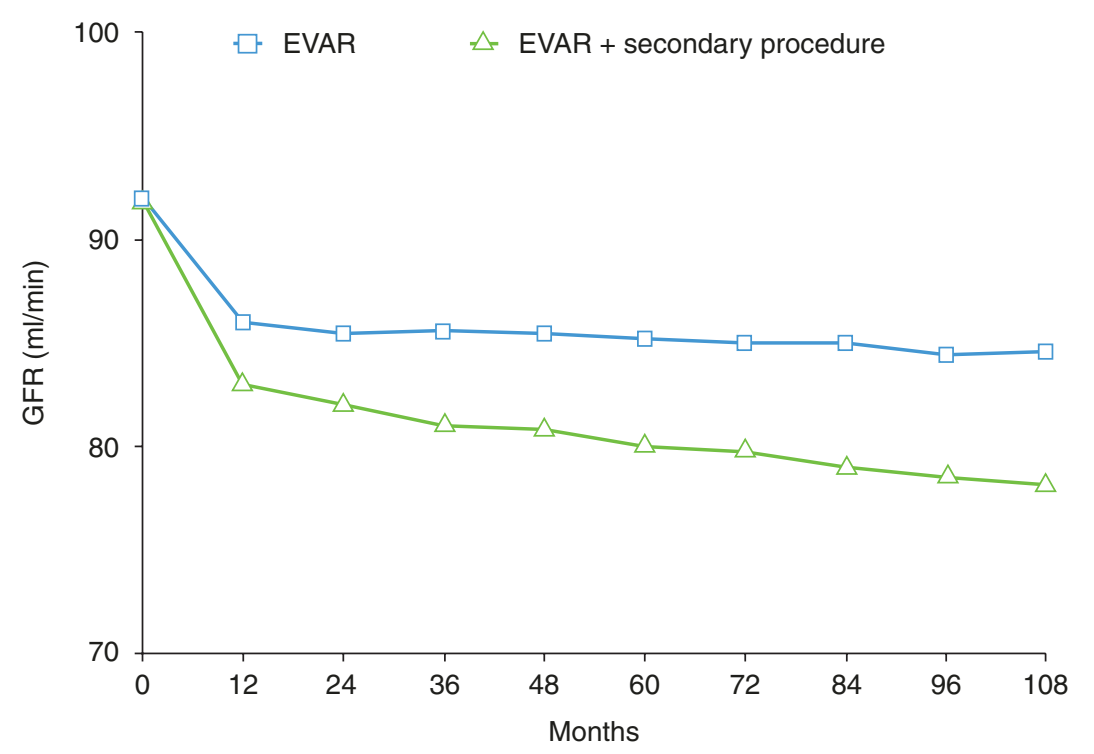

Fig. 6.6 Long-term results on renal function between patients undergoing endovascular aneurysm repair (EVAR) and EVAR with secondary procedure. Glomerular filtration rate (GFR) was estimated through renal perfusion scintigraphy (RPS)

\subsection{Discussion}

Both endovascular and open repairs of AAAs can be associated with postoperative renal function modifications, but the precise role of these procedures especially on long-term renal outcomes is unclear.

In particular, the effects of EVAR on renal function have been questioned since its introduction, but the question still remains unresolved even if it now represents an accepted method of treatment in appropriate candidates with infrarenal abdominal aortic aneurysm. In this context, the introduction of TRF to improve endograft fixation raised adjunctive concerns regarding its effects on renal function.

The comparison of TRF vs IRF has been largely investigated since flow impairment due to bare stents across the ostia of the renal artery may be a contributing factor in renal function decline [17]. Lau et al. investigated the effect of TRF versus IRF on renal function (assessed by $S_{\mathrm{Cr}}$ ) and renal artery patency demonstrating that TRF does not lead to significant renal dysfunction or renal artery occlusion [18]. Mehta et al., in a review of their experience with EVAR on 496 patients (111 with IRF, 385 with TRF), found a significant increase of $S_{\mathrm{Cr}}$ and a decrease on $\mathrm{Cl}_{\mathrm{Cr}}$ over the long-term follow-up in both groups [19, 20]. A meta-analysis on this topic provided conflicting results and concluded that the data were insufficient to draw any strong conclusions on the effect of TRF and IRF on renal function [21]. All these studies were based on the use of $S_{\mathrm{Cr}}$ and $\mathrm{Cl}_{\mathrm{Cr}}$ to assess renal function, with the inherent limitations.

On the other hand, our experience is based on RPS to investigate renal function, since it is highly sensitive, can detect even small alterations of GFR, and allows to investigate the two kidneys separately [22, 23]. Moreover, in our studies, we included only patients without severe preoperative renal failure, which represents a known risk factor for renal postoperative renal complications. In a first pilot study in which we analyzed 47 consecutive patients undergoing EVAR with TRF, GFR impairment was documented at RPS in the $10.6 \%$ of cases [24], but there was no significant difference in postoperative GFR between IRF and TRF.

Another issue regards the long-term renal outcomes. The analysis of data from the large 
trials EVAR 1 and EVAR 2 [29] led to the conclusion that the long-term renal function (estimated by the Modification of Diet in Renal Disease calculation) appears to be stable over time with little evidence to suggest any difference attributable to aneurysm management policies. Our experience, however, demonstrated a marked decline over time in renal function in EVAR compared with OR $(P<0.001)$ that started in the early postoperative period (13\% vs $4 \% ; P<0.001)$ and persisted throughout followup, with a reduction of the GFR at 9 years of $11 \%$ compared with 3\% [25]. This may be related to a combination of CIN and renal atheroembolism. It is noteworthy that the RPS revealed a significant reduction $(>20 \%)$ of the GFR in nine $(6 \%)$ EVAR patients (four with TRF and five with IRF) that was limited to a single kidney that may be ascribed to microembolism during the endovascular procedure, such as manipulation of the endograft within the aortic neck near the renal ostia and balloon expansion of the proximal stent. This result is in line with findings of punctate renal infarctions on follow-up CT scans that have been described in other studies, suggesting a possible embolic sequela after EVAR [18, 30].

The issue of contrast nephropathy could be particularly relevant in patients who required one or more additional endovascular procedure with the follow-up mainly based on contrast-enhanced CT. The results of our study demonstrated a close relation between adjunctive procedures performed in the long term and the decline in renal function. In fact, in the 24 patients $(17 \%)$ that required a supplementary endovascular procedure during follow-up, the decline of the GFR at 9 years was remarkably higher $(P<0.0005)$.

In our series, as well as in other reports, type II endoleak was the main cause of secondary procedures; furthermore, these procedures have a low technical success, and up to $40 \%$ of patients require additional re-intervention during followup [31]. In this scenario, we proposed a modified approach to EVAR consisting in the prevention of type II endoleaks rather than their treatment. This approach, based on aneurysm sac embolization using coil and fibrin glue [31-33], has been dem- onstrated to reduce type II endoleaks and related re-intervention. Furthermore, we have revised our surveillance protocol in order to reduce contrast dye administration during the follow-up that currently is based on abdominal CT (without contrast dye) and Doppler ultrasound. Contrast-enhanced $\mathrm{CT}$ is reserved only in case of aneurysmal sac enlargement, persisting type II endoleak, or signs of endograft migration.

Our study has some limitations that are noteworthy. The small number of patients treated by EVAR (especially if compared with those treated by OR) limits the ability to perform subgroup analysis and to identify statistically significant risk factors for progressive renal function decline. The greater number of ASA IV patients $(P<0.0001)$ in the EVAR groups may influence results on renal function, especially in the long term. However, the greatest advantage of our studies is to have investigated the kidney function by RPS which, in our opinion, overcomes the limitations of the common biochemical markers allowing an accurate assessment of even subtle impairments confined in a single kidney.

Considering all the weaknesses of our experience, it is certainly not possible to draw any strong conclusions about the real nephrological risk of a procedure than another for treatment of AAAs. However, on the basis of our evidence, especially with regard to the GFR impairment after EVAR both with TRF and IRF, it is reasonable to conclude that among the factors that could affect the patient suitability for EVAR, preoperative renal conditions should be taken into account.

\subsection{Perspectives}

With the technological evolution of endografts, fenestrated and branched endografts are becoming an established type of treatment for juxtarenal, pararenal, and thoracoabdominal aneurysms. These types of endografts require higher volume of contrast dye compared to standard EVAR during the procedure; furthermore, they require renal artery cannulation with guidewires and catheters and stent deployment. These maneuvers, along with contrast media administration during follow-up and the risk 
of stent compression or occlusion, may have a role, but data are lacking regarding the early and longterm effects of these types of treatment on renal function. On the other hand, open surgery also carries a non-negligible risk of renal function decrease in the treatment of complex AAAs and thoracoabdominal aneurysms, but a direct comparison between the two techniques is needed.

The use of new technologies may limit the dose of contrast dye used for endovascular procedures. The Magellan robotic system (Hansen Medical, Mountain View, CA, USA) has a catheter technology designed to deliver stability and distal tip control of guidewires and catheters and has been demonstrated to reduce the duration of complex endovascular procedures. These advantages could be associated to a decreased need for contrast media and subsequent reduction of renal complications.

Another interesting field is the use of carbon dioxide $\left(\mathrm{CO}_{2}\right)$ angiography during endovascular procedures, which is associated to a virtually absent risk of renal impairment. This technology is already used in some centers especially for peripheral procedurals, but large data on feasibility and effectiveness in aortic procedures are needed.

Finally, considering that secondary procedures are strictly associated to renal function worsening during follow-up, improvements in the optimization of patient selection and prevention of complications and secondary endovascular procedures are needed. Considering that type II endoleak represents the most frequent cause of re-intervention after EVAR, in our opinion, the use of type II endoleak-preventing procedures at the time of EVAR could be useful.

\section{References}

1. Zarins CK, White RA, Scwarten D, et al. Aneurex stent graft versus open surgical repair of abdominal aortic aneurysms: multicenter prospective clinical trial. J Vasc Surg. 1999;29:292-305.

2. Becquemin JP, Lapie V, JPn F, et al. Mid-term results of a second bifurcated endovascular graft for abdominal aortic aneurysm: the French Vanguard trial. J Vasc Surg. 1999;30:209-18.
3. Ligush J Jr, Pearce JD, Edwards MS, et al. Analysis of medical risk factors and outcomes in patients undergoing open versus endovascular abdominal aortic aneurysm repair. J Vasc Surg. 2002;36:492-9.

4. Veith FJ, Johnston KW. Endovascular treatment of abdominal aortic aneurysms: an innovation in evolution and under evaluation. J Vasc Surg. 2002;35:183.

5. Wartman SM, Woo K, Yaeger A, et al. Outcomes after abdominal aortic aneurysm repair requiring a suprarenal cross-clamp. J Vasc Surg. 2014;60(4):893-9.

6. Sternbergh WC III, Carter G, York JW, et al. Aortic neck angulation predicts adverse outcome with endovascular abdominal aortic aneurysm repair. J Vasc Surg. 2002;35:482-846.

7. Chaikof EL, Fillingar MF, Matsumura JS, et al. Identifying and grading factors that modify the outcome of endovascular aortic aneurysm repair. J Vasc Surg. 2002;35(5):1061-6.

8. Becker GJ, Kovacs M, Mathison MN. Opinion: Transluminal repair of abdominal aortic aneurysm: a call for selective use, careful surveillance, new device design and systematic study of transrenal fixation. J Vasc Surg. 2002;35:611-5.

9. Malina M, Brunkwall J, Ivancev K, et al. Renal artery covered by aortic stents: clinical experience from endovascular grafting of aortic aneurysms. Eur J Vasc Endovasc Surg. 1997;14:109-13.

10. Duda SH, Raygrotzki S, Wiskirchen J, et al. Abdominal aortic aneurysms: treatment with juxtarenal placement of covered stent-grafts. Radiology. 1998;206:195-8.

11. Marin ML, Parsons RE, Hollier LH, et al. Impact of transrenal aortic endograft placement on endovascular graft repair of abdominal aortic aneurysms. J Vasc Surg. 1998;28:638-46.

12. MacIerewicz J, Walker SR, Vincent R, et al. Vascular Surgical Society of Great Britain and Ireland: perioperative renal function following endovascular repair of abdominal aortic aneurysm with suprarenal and infrarenal stents. Br J Surg. 1999;86:696.

13. Bove PG, Long GW, Zelenock GB, et al. Transrenal fixation of aortic stent-grafts for the treatment of infrarenal aortic aneurismal disease. J Vasc Surg. 2000;32:697-703.

14. Lobato AC, Quick RC, Vaugh PL, et al. Transrenal fixation of aortic endografts: intermediate follow-up of a single-center experience. $\mathrm{J}$ Endovasc Ther. 2000;7:273-8.

15. Kichikawa K, Uchida H, Maeda M, et al. Aortic stent-grafting with transrenal fixation: use of newly designed spiral Z-stent endograft. J Endovasc Ther. 2000;7:184-91.

16. Izzedine H, Koskas F, Cluzel P, et al. Renal function after aortic stent-grafting including coverage of renal arterial ostia. Am J Kidney Dis. 2002;39:740-36.

17. Birch PC, Start RD, Whitbread T, et al. The effects of crossing porcine renal ostia with various endovascular stents. Eur J Vasc Endovasc Surg. 1999;17:185-90.

18. Lau LL, Hakaim AG, Oldenburg WA, et al. Effect of suprarenal versus infrarenal aortic endograft fixation on renal function and renal artery patency: a compara- 
tive study with intermediate follow-up. J Vasc Surg. 2003;37:1162-8.

19. Cayne NS, Rhee SJ, Veith FJ, et al. Does transrenal fixation of aortic endografts impair renal function? J Vasc Surg. 2003;38(4):639-44.

20. Mehta M, Cayne N, Veith FJ, et al. Relationship of proximal fixation to renal dysfunction in patients undergoing endovascular aneurysm repair. J Cardiovasc Surg. 2004;45(4):367-74.

21. Walsh SR, Boyle JR, Lynch AG, et al. Suprarenal endograft fixation and medium-term renal function: systematic review and meta-analysis. J Vasc Surg. 2008;47:1364-70.

22. Petersen LJ, Petersen JR, Talleruphuus U, et al. Glomerular filtration rate estimated from the uptake phase of ${ }^{99 \mathrm{~m}} \mathrm{Tc}-\mathrm{DTPA}$ renography in chronic renal failure. Nephrol Dial Transplant. 1999; 14:1673-8.

23. Awad RW, Barham WJ, Taylor DN, et al. The effect of infrarenal aortic reconstruction on glomerular filtration rate and effective renal plasma flow. Eur J Vasc Surg. 1992;6:362-7.

24. Deriu GP, Grego F, Lepidi S. Short-term arterial blood reperfusion of normothermic kidney in renal artery and abdominal aorta reconstructive surgery. Eur J Vasc Endovasc Surg. 2001;21(4):314-9.

25. Grego F, Frigatti P, Antonello M, et al. Suprarenal fixation of endograft in abdominal aortic aneurysm treatment: focus on renal function. Ann Surg. 2004;240(1):169-78.
26. Maeder M, Klein M, Fehr T, et al. Contrast nephropathy: review focusing on prevention. J Am Coll Cardiol. 2004;44(9):1763-71.

27. Antonello M, Menegolo M, Piazza M, et al. Outcomes of endovascular aneurysm repair on renal function compared with open repair. J Vasc Surg. 2013;58(4):886-93. https://doi.org/10.1016/j. jvs.2013.02.249. Epub 2013 May 17.

28. Chaikof EL, Blankensteijn JD, Harris PL, et al. Reporting standard for endovascular aortic aneurysm repair. J Vasc Surg. 2002;35:1048-60.

29. Brown LC, Brown EA, Greenhalgh RM, et al. Renal function and abdominal aortic aneurysm. The impact of different management strategies on long-term renal function in the UK endovascular aneurysm repair (EVAR) trial. Ann Surg. 2010;251:966-75.

30. Kramer SC, Seifarth H, Pamler R, et al. Renal infarction after endovascular aortic aneurysm repair. J Endovasc Ther. 2002;9:98-102.

31. Abularrage CJ, Patel VI, Conrad MF, et al. Improved results using Onyx glue for the treatment of persistent type 2 endoleak after endovascular aneurysm repair. J Vasc Surg. 2012;56:630-6.

32. Zanchetta M, Faresin F, Pedon L, et al. Intraoperative intrasac thrombin injection to prevent type II endoleak after endovascular abdominal aortic aneurysm repair. J Endovasc Ther. 2007;14:176-83.

33. Piazza M, Frigatti P, Scrivere P, et al. Sac embolization during EVAR to prevent type II endoleak: preoperative aneurysm volumetric analysis as predictor of efficacy. J Vasc Surg. 2013;57:934-41. 